\title{
Reflets
}

Revue ontaroise d'intervention sociale et communautaire

\section{Transiger quotidiennement avec des phénomènes de violence : risque personnel pas toujours calculé}

\section{Léo Dubord}

Volume 2, numéro 1, printemps 1996

Contrer la violence subie par les femmes et les enfants en milieu familial

URI : https://id.erudit.org/iderudit/026110ar

DOI : https://doi.org/10.7202/026110ar

Aller au sommaire du numéro

Éditeur(s)

Reflets : Revue ontaroise d'intervention sociale et communautaire

ISSN

1203-4576 (imprimé)

1712-8498 (numérique)

Découvrir la revue

Citer cet article

Dubord, L. (1996). Transiger quotidiennement avec des phénomènes de violence : risque personnel pas toujours calculé. Reflets, 2(1), 134-145. https://doi.org/10.7202/026110ar
Résumé de l'article

Cet article présente la problématique de la travailleuse sociale quitransige quotidiennement avec des phénomènes de violence. Ondémasque certains messages subtils cachés sous la plupart des actesviolents. Enfin, l'auteur propose des méthodes concrètes pourcontrer les raisonnements qui maintiennent subrepticement leclimat de violence.
Tous droits réservés (C) Reflets : Revue ontaroise d'intervention sociale et communautaire, 1996
Ce document est protégé par la loi sur le droit d'auteur. L'utilisation des services d'Érudit (y compris la reproduction) est assujettie à sa politique d'utilisation que vous pouvez consulter en ligne.

https://apropos.erudit.org/fr/usagers/politique-dutilisation/ 


\section{Transiger quotidiennement avec des phénomènes de violence: risque personnel pas toujours calculé $^{1}$}

\section{Léo D ubord}

$P$ sychothérapeute familial au $C$ entre $U$ vannik, 0 ttawa

P rofesseur de psychothérapie conjugale, U niversité Saint-Paul, 0 ttawa

C et article présente la problématique de la travailleuse sociale qui transige quotidiennement avec des phénomènes de violence. $0 \mathrm{n}$ démasque certains messages subtils cachés sous la plupart des actes violents. Enfin, l'auteur propose des méthodes concrètes pour contrer les raisonnements qui maintiennent subrepticement le climat de violence.

\section{La problématique}

Les actes de violence conjugale, familiale et sociale les plus scabreux, ou parfois les plus surnois, confrontent quotidiennement la travailleuse qui oeuvre dans le domaine de la santé mentale. Q uel impact une telle sur-exposition à la férocité humaine peutelle produire à plus ou moins long terme sur le bien-être de ce personnel dévoué? 
Il est relativement facile de mesurer l'impact d'une surexposition à des radiations nucléai res comme dans le cas de C hernobyl, parce qu'il reste des marques physiques. Les marques psychologiques se mesurent beaucoup plus difficilement, car elles tendent à disparaître peu de temps après le choc, puis à réapparaitre plusieurs années plus tard sous des formes presque totalement imprévisibles.

D ans cet article, nous maintenons qu'une surexposition à la violence sousquelque forme que ce soit entraîne des conséquences dépassant de loin les phénomènes de burnout déjà bien connus danslesmilieux spécialisés en santé mentale. $\mathrm{N}$ ousallons expliquer que cette violence crée des sentiments d'impuissance professionnelle et personnelle, diminue la créativité et obstrue la formation d'idées nouvelles permettant de trouver de nouvelles solutions à de vieux problèmes.

\section{Messages subtils}

U n message subtil est une communication tellement imperceptible qu'elle est souvent considérée comme quantité négligeable dans les protocoles d'étude d'impact d'un phénomène sur l'autre. Par exemple, il était de mise jadis de frapper lesélèves dans les écoles. Jusqu'à une date récente, personne n'avait pensé que ces traitements induisaient chez les jeunes des états de révolte, de nervosité, de dégoût des études, etc. C ette manière d'imposer la discipline communiquait insidieusement au jeune plusieurs messages: «es adultes ne te comprennent pas!» «N e fais pas confiance aux adultes!» «Tu n'es pas capable d'apprendre; tu es trop bête!» etc.

D'autres messages ont de plus la propriété de produire des effets cumulatifs, qui, à force de s'accumuler, produisent des résultats inimaginables. L'exemple le mieux connu est celui du peloton de soldats qui marchent au pas sur un pont. La cadence répétée de leurs pas peut faire crouler le pont. 0 u encore, la voix 
de la soprano qui fracasse un verre, à cause de la percussion répétée des vibrations sonores.

Les mathématiciens modernes ont donné le nom «d'effet papillon $»^{2}$ à ce phénomène (accumulation de petites poussées qui produisent des effets inattendus). 0 n nous fait imaginer le scénario suivant: un maringouin s'envole du dos d'un boeuf australien après s'être repu de son sang. Le battement de ses ailes cause une légère perturbation de l'air environnant. Si d'aventure, papillons et autres insectes agitent aussi de leurs ailes ce même air environnant, et si ensuite des oiseaux multiplient cette agitation, si de petits avions et ensuite de gros quadrimoteurs augmentent le brassement d'air à un degré assez élevé, nous aboutirons, paraitil, à un ouragan en Californie six mois plus tard!

$D$ ans son très beau livre Imagery for $G$ ettingW ell, D eirdre $D$ avis Brigham (1994) nous raconte un phénomène intriguant, qui démontre la réalité des communications subtiles. Elle appelle son observation $L a$ théorie du C entième macaque. La scène se passa au Japon, alors que des chercheurs faisaient une étude sur les macaques. Les chercheurs donnaient à leurs sujets d'expérience des tranches de pommes de terre. Bien que prisant les pommes de terre, les singes aimaient moins le gravier qui adhérait aux tranches déposées sur le sol. U n jour, soit par hasard, soit par ingéniosité, un macaque réalisa qu'en mettant sa tranche dans l'eau, le gravier disparaissait. II se mit donc à laver régulièrement sa nourriture.

Les savants remarquèrent alors que tous les deux ou trois jours, un autre singe apprenait, par imitation, à laver sa nourriture. C e rythme d'apprentissage se maintint pendant assez longtemps. M ais lorsqu'un nombre suffisamment grand de singes connut la manoeuvre, soudain, le lendemain, tous les singes de la tribu savaient laver leur nourriture! Q uel était ce phénomène? Existe- $t$ - $i l$ une atmosphère qui permette des communications subtiles, lorsqu'un nombre suffisamment grand d'individus fait quelque chose? Si tel est le cas, l'avantage est énorme lorsque l'apprentissage est positif. $M$ ais s'il est négatif, le désavantage est certainement catastrophique. 
Si ces communications subtiles existent, nous posons de nouveau notre question du début: qu'arrive-t-il au bien-être des intervenantes familiales et sociales qui transigent quotidiennement, à tous les niveaux, avec la violence conjugale et familiale?

\section{Message subtil le plus puissant de la violence: «tu n'y peux rien»}

Q ue la violence soit d'ordre physique ou psychologique, elle contient toujours le même message destiné à la victime: «Tu es plus faible que moi; tu ne me vaincras pas; tu n'y peux rien; tu dois endurer mes sévices?. N ousne pourrion simaginer de message plus destructeur, signifiant que la victime ne possède plus ni la force de se défendre ni la faculté de penser. $C$ 'est une forme d'aliénation, d'anéantissement.

Les travailleuses sociales qui ont rencontré des victimes de sévices sexuels, physiques ou autres, constatent souvent cette aliénation chez leurs clientes. Par contrecoup, on accuse les victimes d'être stupides ou niaiseuses, de ne pas être motivées pour améliorer leur sort. Ce contre-transfert est d'autant plus facilement provoqué que la victime dira souvent, de son agresseur, avec un air de découragement: «e l'aime encore!»

Si une travailleuse sociale éprouve facilement un contretransfert négatif vis-à-vis de ses clientes, nous proposons l'idée qu'elle a subi, dans son travail quotidien, l'influence négative des messages subtils de la violence. Si elle devient facilement désabusée et négative devant les rechutes répétées des victimes, elle aussi a subi l'influence négative des messages subtils de la violence dans son travail. N ous affirmions plus haut qu'une des influences négatives de la violence est de détruire la créativité et les idées nouvelles. $\mathrm{N}$ ous allons donc proposer des idées pour favoriser le renouvellement professionnel et personnel. 


\section{Que faire lorsqu'une femme affirme aimer son persécuteur?}

La première réponse à cette question est: ne pas se laisser envahir par l'impuissance. Les lignes qui suivent indiquent comment.

II faut d'abord faire une constatation générale. Le processus par lequel, en tant qu'être humain, on en arrive à une conclusion comme «je l'aime encore» est le suivant: nos sens transmettent à notre cerveau une sensation, sous la forme d'une vibration électrique. Par conditionnement environnemental, nous interprétons cette sensation, que nous traduisons par des mots alignés pour former des phrases.

L'interprétation donnée à ces sensations est donc largement subjective, et conditionnée par l'environnement. Si jamais nous recevons des sensations que nous ne savons pas interpréter, ou que l'environnement ne nous a pas habituées à interpréter d'une façon qui expliquerait notre comportement, nous avons recours à des interprétations apprises à l'avance, même si elles ne cadrent pas avec précision avec la réalité que nous vivons.

La femme victime de violence fait probablement, à son insu, ce genre de manoeuvre. Elle reçoit des coups ou subit des sévices psychologiques qu'elle ressent comme désagréables. Comme toute personne saine, elle a besoin de s'expliquer à elle-même le fait qu'elle continue de vivre avec le conjoint violent. Comme elle ne trouve pas d'explications cohérentes, elle a recours à l'explication classique: «e l'aime encore!».

Si la travailleuse sociale comprend ces processus psychologiques, elle ne se fâchera pas contre la cliente qui explique ainsi son comportement. Elle l'aidera plutôt de la manière suivante: «A u moment où il vous frappe, est-ce que vous l'aimez? Au moment où vous ressentez la douleur du coup, est-ce que vous l'aimez? Lorsqu'il entre à la maison sans même vous regarder et qu'il s'asseoit devant le téléviseur avec sa bière, est-ce que vous l'aimez? Q uand il est agressif avec vous au moment de faire I'amour, est-ce que vous l'aimez?» II est probable que la personne 
répondra par la négative à toutes ces questions. A lorsl'intervenante sociale peut rétorquer: «M ais alors, quand l'aimezvous?»

C ette remarque introduira le doute dans les explications de la victime. Si elle reprend «qu'il a des qualités», la psychothérapeute peut répondre: «Évidemment, il doit avoir de grandes qualités, vous I'avez épousé! M aintenant, est-ce que les coups que vous recevez valent ses qualités?» D e cette façon, l'intervenante sociale a déjà situé la discussion sur un autre plan, qui permet à la victime de prendre du recul émotif vis à-vis de son conjoint. La thérapeute peut alors passer en revue les qualités du conjoint par rapport à ses défauts (car désormais il peut avoir des défauts), et la cliente peut utiliser de meilleurs critères pour évaluer les qualités qu'elle peut exiger de son mari.

Grâce à cette méthode, ni la victime ni la travailleuse sociale ne sont dans l'impuissance créée par la violence. A insi, «Elles n'y peuvent rien!» devient «Elles y peuvent quelque chose!».

Sur le plan professionnel, les possibilités d'aider une victime de la violence, à quelque niveau que ce soit, sont infinies. Si par exemple une femme, à la suite d'agression sexuelle, est hantée par des images traumatisantes ou des cauchemars répétitifs, la psychothérapeute peut procéder comme suit: elle fait raconter l'événement en détail. C e travail doit se faire lentement et avec tact. S'il faut quatre ou six heures pour amener une personne à raconter un événement traumatisant, il ne faut pas s'en formaliser. Lorsque la victime réussit à raconter l'événement, la thérapeute la fait revenir sur le moment qui semble évoquer le plus de réactions émotives. Puis elle dit à sa cliente: «e pense qu'il faudrait explorer plus loin. Êtes-vous d'accord?» II est peu probable que cette dermière offre beaucoup de résistance à ce moment, car le terrain a été bien préparé. L'intervenante peut alors lui demander beaucoup de détails physiques inattendus: «Vous étiez devant votre auto; du côté de la porte de la conductrice? Aviez-vous votre sac à main? Accroché à quel bras? De quelle couleur est votre voiture? Est-ce une voiture à deux portes? L'agresseur vous a d'abord touché quelle épaule?» etc. Bien que pénibles au début, ces détails ont pour but de démystifier les circonstances et de les situer dans le concret. 
Lorsque les circonstances sont démystifiées, la thérapeute fait le même travail d'exploration sur le plan émotif: quelle fut sa première réaction émotive en réalisant ce qui se produisait? les idées qui lui sont venues en tête? ce qu'elle a tenté de faire? avec quel résultat? Pendant cette exploration, la travailleuse sociale cherche les moments d'impuissance. Son but est de réintroduire le sentiment de puissance à tous les endroits où il a été détruit. C ela ne peut se faire que si la victime ressent une empathie totale avec sa thérapeute. Si cette dernière est envahie par trop de révolte ou de colère vis-à-vis de l'événement, la cliente le percevra, et il n'est pas certain qu'elle pourra se distancier du traumatisme. Si la travailleuse sociale est assaillie par le sentiment d'être seule dans sa lutte contre la violence, la cliente le percevra, et elle aussi commencera à se sentir abandonnée.

Les victimes vibrent comme des cordes de violon quand elles perçoivent les messages émotifs transmis plus ou moins consciemment par leur thérapeute. U n traumatisme est une condition dans laquelle une personne reste figée et hypersensible pour s'assurer que le monde extérieur ne la blessera pas à nouveau. Pour se protéger, elle observe donc continuellement ce qui se passe autour d'elle.

Sur le plan professionnel, pour une travailleuse sociale l'antidote à la violence est donc la puissance, définie comme la condition que dans les pires situations, il reste toujours quelque chose de nouveau à faire, à penser, à dire, à imaginer, pour remédier à un problème. Lorsqu'il ne reste plus rien à imaginer ou à dire devant un problème, nous avons affaire au burnout, à la dépression, et à la colère.

\section{La santé psychologique de la travailleuse sociale}

N ous sommes d'avis que les femmes sont particulièrement vulnérables aux sensations d'impuissance dans leurs relations avec les hommes, car elles sont physiquement plus petites qu'eux, et elles 
n'ont pas la même morphologie musculaire. Leur grande quantité de muscles lisses les rend plus aptes à faire un effort soutenu qu'à donner un coup inattendu. Les hommes sont mieux équipés pour déployer une force brutale, intense et de courte durée. $O r$, la violence est brutale et de courte durée. Les femmes se sentent donc inaptes à se défendre dans de telles situations.

II faut aussi ajouter que la culture n'a jamais enseigné aux femmes qu'elles avaient autant de pouvoir qu'un homme. À poids égal, et à condition physique égale, les forces tendraient à s'équilibrer. $M$ ais culturellement, les femmes développent peu leur force physique et leur coordination. Elles n'ont commencé que récemment à augmenter leur musculature. Les hommes, au contraire, ont un «moi» construit autour de la force physique. Les femmes ont un «moi» construit autour de la vie émotive ou affective. N ous pouvons rêver d'un monde où le «moi» se construirait autour de l'intelligence.

Q ue peut faire une travailleuse sociale pour s'assurer de ne pas tomber dans le sentiment d'impuissance provenant des messages inhérents à la violence qu'elle rencontre au quotidien dans sa vie professionnelle? N ous proposons ici quelques solutions:

\section{S'assurer qu'elle détecte la violence sous ses formes les plus subtiles ${ }^{3}$}

Cette assertion semble simple, mais n'est peut-être pas aisée à mettre en pratique.Voici un exemple des subtilités culturelles qui nous empêchent de voir ou détecter la violence. L'an dernier, le téléroman le plus suivi au Q uébec était L es filles de C aleb, d'A rlette C outure. $M$ a femme et ma fille étai ent clouées devant le téléviseur. À la troisième émission, je dis à mon épouse que je ne pouvais regarder cette émission, car je la trouvais trop violente. Je lui demandai même si notre fille de treize ans devait regarder cette émission. Elle fut très surprise de ma remarque, mais je cessai d'écouter l'émission.

Devant sa surprise, je me mis à réfléchir que l'émission me plaçait face à moi-même, en tant qu'homme, et me froçait de voir les dégâts accomplis par d'autres hommes. En d'autres 
termes, se pouvait-il que je sois placé face à ma propre violence, que je ne voulais pas la voir? Ce questionnement fut pour moi une source d'inquiétude pendant des semaines. Je ne trouvais toujours pas de réponse, et je ne pouvais toujours pas regarder l'émission.

Je me mis donc à écouter les conversations entre femmes à I'hôpital psychiatrique où je travaille à temps partiel. Je m'étonnai alors de ne jamais entendre de commentaires concernant la violence psychologique décrite dans l'émission. Les gens sympathisaient avec Émilie, mais on ne parlait pas de la violence qu'elle subissait. Lorsque je fis remarquer mon étonnement, leur réponse me sidéra: «Vyons! Tu sais bien que les hommes sont comme ça, et on les aime quand même! Le programme ne décrit que la réalité!» Je commençai donc de m'expliquer mon incapacité à regarder l'émission par ma réaction de désar roi devant l'apathie sociétale contre la violence. N ous ne la voyons plus! $N$ ous la considérons comme «normale». $O r$, sans rejeter l'interprétation (freudiennne) selon laquelle je suis confronté à ma propre violence, je désirerais qu'on la voie où elle se trouve.

\section{Existe-t-il de la violence dans ma vie?}

La vie quotidienne est faite d'événements expliqués par des stéréotypes. Par exemple, nous lisons dans tous les livres portant sur le couple, et nous entendons à toutes les émissions consacrées à la vie conjugale ou familiale, qu'il est «normal» d'avoir des chicanes de couple ou de famille. Est-ce bien «normal»? J'enseigne à des étudiants universitaires la thérapie conjugale. Lorsque je leur parle de mon modèle de couple qui propose «chicane 0 » entre conjoints, ils me traitent d'idéaliste! La chicane fait partie de la vie de couple.

Si nouslisons la définition du Petit R obert au mot «violence», nous voyons ceci : «Agir sur quelqu'un ou le faire agir contre sa volonté, en employant la force ou l'intimidation». Les chicanes ne sont-elles pas des manoeuvres par lesquelles nous tentons, par intimidation, pression ou habileté, à imposer notre volonté à la personne avec laquelle nous nous chicanons? Q ui gagne dans une 
chicane? N e vaudrait-il pas mieux discuter dans un cadre autre que la chicane. La plupart du temps, après une chicane, une des deux personnes est plus blessée que l'autre. C haque blessure érode la capacité de régler les conflits de façon intelligente et équitable.

Ai-je parfois recours à la colère pour faire obéir mes enfants? En soi, la colère n'est pas mauvaise. $M$ ais si je l'utilise pour faire plier la volonté d'une autre personne ou d'un enfant, je verse dans l'intimidation. Dans les enjeux parentaux, la colère peutêtre utilisée pour faire cesser les agissements d'un enfant. Je me rapproche de l'enfant et je l'aide à réfléchir à ce qu'il devrait choisir pour son mieux-être et celui de son entourage. Aider à réfléchir est essentiel. Je ne force pas l'enfant à cesser son comportement. Je l'arrête, je l'aide à réfléchir pour lui permettre de maîtriser ses pulsions à son avantage, ainsi qu'à celui de son entourage.

\section{Évaluer nos perceptions}

N ous sommes toutes prisonnières de notre culture, de nos préjugés, de notre éducation, de nos idéaux. D ernièrement, je recevais en thérapie une femme qui me raconta l'incident suivant: son mari était tout à fait opposé à l'idée que leur fille de dix-huit ans fume. Pour montrer sa détermination, il avait dit à l'adolescente à un moment donné: « $\lesssim i$ je te prends la cigarette au bec, je te casse les dents dans la bouche!» Sa fille avait rapporté l'incident à sa mère. Je lui demandai ce qu'elle avait répondu. Elle me répondit qu'elle ne pouvait rien dire à sa fille, parce qu'elle ne voulait pas détruire le respect qu'elle devait à son père, mais qu'elle avait dit à son conjoint de ne jamais porter la main sur leur enfant. Je lui répondis que ce n'était pas assez. Elle aurait dû conseiller à sa fille: « La prochaine fois, disà ton père que s'il te menace encore, tu déposeras une plainte judiciaire contre lui!» La femme me répondit: «Ça ne se fait pas!» Je lui fis remarquer que si un collègue de travail lui faisait la même menace, elle déposerait une plainte contre lui. Pourquoi protéger un conjoint ou un père plus qu'un collègue?

Selon les perceptions de ma cliente, on ne peut faire intervenir le 
système judiciaire dans une dispute familiale. $\mathrm{O} u$ encore, on ne le fait intervenir que si «c'est très grave!» Est-ce que ce n'est pas «très grave» de recevoir la menace de se faire casser les dents? Évidemment, je ne propose pas de faire constamment intervenir le système judiciaire dans les disputes familiales. $M$ ais le message que doit recevoir celui qui menace et intimide est: «e ne suis pas impuissante; j'ai les moyens de me défendre!»

Les échanges et la discussion sont nécessaires pour donner du recul à notre manière d'évaluer les actes de violence. $D$ ans les relations affectives importantes, et toutes les relations familiales sont importantes, nous devons constamment réévaluer nos critères de jugement. Ce qui existe depuis toujours se normalise et ne provoque plus de réactions. Les formes de violence peuvent se glisser dans notre vie quotidienne sans que nous ne les voyions. $M$ ais si à un moment donné, nous nous demandons pourquoi nous n'avons «plus le goût à rien», «pourquoi plus rien ne nous tente», il se pourrait qu'il existe des formes subtiles de violence dans notre vie quotidienne.

\section{Se fier à nos sensations.}

Comme nous le disions plus haut, nos sens apportent à notre cerveau des messages sous forme de pulsations bioélectriques. $\mathrm{N}$ ous interprétons ensuite ces pulsations en termes émotifs. Concrètement, chaque fois que dans une interaction nous commençons à nous sentir mal à l'aise, il est important de nous arrêter pour vérifier ce qui se passe. II peut s'agir de violence subtile. N ous ne voulons pas toujours confronter les personnes qui peuvent nous transmettre des messages violents. $M$ ais nous gardons, bien rodées, nos aptitudes à l'interprétation à utiliser en cas de besoin. La conscience de ce qui se passe est une arme efficace de prévention contre les sensations d'impuissance causées par les messages subtils de violence.

En conclusion, nous pourrions discourir longuement sur la problématique de la travailleuse sociale qui quotidiennement transige avec des personnes victimes de violence conjugale. Par cet 
article, nous avons tenté de soumettre quelques pistes qui pourraient alimenter la réflexion. Pour nous, les rapports «nor maux» sont faits de bonne humeur, de bonne entente, de discussions agréables, non de chicanes, de remarques abaissantes ou d'assertion exprimant les préjugés d'un sexe contre l'autre.

\section{Notes}

1. Les idées exprimées dans cet article furent originellement proposées dans le cadre d'une conférence donnée aux travailleuses sociales de la région $\mathrm{H}$ ull, Aylmer et Gatineau, sous le titre: I mpact sur les intervenantes, lorsque confrontées aux phénomènes de la violence, de la pauvreté et du multialturalisme. Cette conférence fut publiée dans sa version originale dans le bulletin de l'A ssociation des psychothérapeutes conjugaux et familiaux du Q uébec, vol. 22, no 3, automne 1995.

2. «L'effet papillon» s'inscrit dans le cadre de la théorie du chaos, vulgarisée par James G leick (1987). La référence précise de ce livre paraît dans la bibliographie.

3. Jusqu'à la fin de notre article nous utiliserons le 〈je», car il s’agit d'exemples plus personnels.

\section{Bibliographie}

DAVIS BR IGHAM Deirdre, M.A. (1994), Imagery for G etting W ell. W.W. N ew York, N orton et Company.

GLEICK, James (1987), C haos, M aking A new Science. N ew York, Penguin Books:N ew York. 